\title{
Optical imaging of hemoglobin oxygen saturation using a small number of spectral images for endoscopic application
}

Takaaki Saito

Hiroshi Yamaguchi

\section{SPIE.}




\title{
Optical imaging of hemoglobin oxygen saturation using a small number of spectral images for endoscopic application
}

Takaaki Saito* and Hiroshi Yamaguchi

Fujifilm Corporation, Imaging Technology Center, Miyanodai 798, Kaisei, Kanagawa 258-8538, Japan

\begin{abstract}
Tissue hypoxia is associated with tumor and inflammatory diseases, and detection of hypoxia is potentially useful for their detailed diagnosis. An endoscope system that can optically observe hemoglobin oxygen saturation $\left(\mathrm{StO}_{2}\right)$ would enable minimally invasive, real-time detection of lesion hypoxia in vivo. Currently, point measurement of tissue $\mathrm{StO}_{2}$ via endoscopy is possible using the commercial fiber-optic oximeter T-Stat, which is based on visible light spectroscopy at many wavelengths. For clinical use, however, imaging of $\mathrm{StO}_{2}$ is desirable to assess the distribution of tissue oxygenation around a lesion. Here, we describe our $\mathrm{StO}_{2}$ imaging technique based on a small number of wavelength ranges in the visible range. By assuming a homogeneous tissue, we demonstrated that tissue $\mathrm{StO}_{2}$ can be obtained independently from the scattering property and blood concentration of tissue using four spectral bands. We developed a prototype endoscope system and used it to observe tissue-simulating phantoms. The $\mathrm{StO}_{2}(\%)$ values obtained using our technique agreed with those from the T-Stat within $10 \%$. We also showed that tissue $\mathrm{StO}_{2}$ can be derived using three spectral band if the scattering property is fixed at preliminarily measured values. $\odot$ The Authors. Published by SPIE under a Creative Commons Attribution 3.0 Unported License. Distribution or reproduction of this work in whole or in part requires full attribution of the original publication, including its DOI. [DOI: 10.1117/1.JBO.20.12.126011]
\end{abstract}

Keywords: hemoglobin oxygen saturation; endoscopy; hypoxia imaging; tissue-simulating phantom.

Paper 150481RR received Jul. 16, 2015; accepted for publication Nov. 30, 2015; published online Dec. 23, 2015.

\section{Introduction}

An imbalance in oxygen supply and demand causes hypoxia in solid tumor tissues, and tumor cells in chronic hypoxia show resistance to radiotherapy and chemotherapy, which leads to a poor prognosis for cancer patients. ${ }^{1-4}$ The treatment-resistant property of the tumor cells is a result of their adaptive response to the hypoxic environment by regulating oxygen-dependent gene expression. Recent studies have shown that hypoxia is also related to inflammation. ${ }^{5,6}$ Inflamed lesions can often become severely hypoxic, and the oxygen-dependent metabolism is also involved in inflammatory processes. Thus, detecting tissue hypoxia can provide useful information for detailed differential diagnosis and selection of therapeutic strategies of cancerous and inflammatory lesions.

Since the hemoglobin oxygen saturation $\left(\mathrm{StO}_{2}\right)$ varies with the dissolved oxygen concentration $\left(\mathrm{dO}_{2}\right)$ in a tissue, and the absorption spectrum of the hemoglobin also varies with its $\mathrm{StO}_{2}$, hemoglobin has often been used for optical measurements of tissue oxygen level. The tissue oximeter T-Stat (Spectros Co., Portola Valley) is a representative tool for measuring tissue $\mathrm{StO}_{2}{ }^{7}{ }^{\mathrm{T}} \mathrm{T}-\mathrm{Stat}$ has several types of probes and enables singlepoint measurement of tissue $\mathrm{StO}_{2}$ in vivo by illuminating tissue with white light and then analyzing the reflectance spectrum in the visible range. The performance of T-Stat has been validated by tissue-simulating phantom and animal experiments, and also by some human studies. ${ }^{7-9}$ Measurement of tissue oxygenation may provide useful information for endoscopic diagnosis, and

*Address all correspondence to: Takaaki Saito, E-mail: takaaki.saito@fujifilm .com there have been some clinical studies using T-Stat where the $\mathrm{StO}_{2}$ of gastrointestinal tissues was endoscopically measured. ${ }^{10,11}$ However, endoscopic application of spectroscopybased oximetry has the following problems: (1) although oxygen concentration can be distributed heterogeneously in a cancerous lesion, ${ }^{12}$ single-point measurement cannot provide the spatial information; and (2) capturing images of many wavelengths for reflectance spectrum analysis is time-consuming, and the results are often blurred because the target tissue does not remain in a state of rest under endoscopic observation.

Several studies have used the hyperspectral imaging method for in vivo optical imaging of $\mathrm{StO}_{2}$ distribution, using multiple images corresponding to different light wavelengths. ${ }^{13,14}$ However, very few studies have reported on $\mathrm{StO}_{2}$ imaging using a small number of wavelengths. As an application for a fundus camera, Nakamura et al. ${ }^{15}$ presented an $\mathrm{StO}_{2}$ imaging technique for human retinal vessels using only two wavelengths: 545 and $560 \mathrm{~nm}$. However, this method focuses on the simple structure of the retinal tissue, where thick blood vessels sparsely exist near the surface. For endoscopic application, the imaging target should be thin vessels embedded in a tissue with strong light scattering. Nadeau et al. ${ }^{16}$ exploited the technique of spatial frequency domain imaging (SFDI) using three wavelengths of 658,730 , and $850 \mathrm{~nm}$ to visualize $\mathrm{StO}_{2}$ distribution of porcine kidneys. However, SFDI needs spatially modulated illumination in principle, and it is impractical to apply the technique to common forward-viewing endoscopes.

We are currently developing an $\mathrm{StO}_{2}$ imaging technique for endoscopic application using a small number of wavelength ranges of light reflected from tissue, focusing on esophageal, gastric, and colorectal mucosa as the target tissues. We 
previously developed an $\mathrm{StO}_{2}$ imaging technique based on three wavelength ranges and applied it to a laser illumination endoscope system. ${ }^{17}$ Here, to argue for the application of $\mathrm{StO}_{2}$ imaging to clinical endoscopy, it is important to show that tissue $\mathrm{StO}_{2}$ can be obtained by a small number of wavelengths equivalently to a spectroscopy-based technique using many wavelengths. In this work, the physical principle of our method based on tissue optics is explained. We verified this method by observing tissue-simulating phantoms whose $\mathrm{dO}_{2}$ was controlled.

\section{Principle of $\mathrm{StO}_{2}$ Calculation}

To derive tissue $\mathrm{StO}_{2}$ using a small number of spectral bands, selection of the appropriate wavelength and bandwidth of each band is essential. The algorithm of our technique employs the following four wavelength ranges: two blues (narrow bands around 450 and $470 \mathrm{~nm}$ ), green (broad band at 500$560 \mathrm{~nm}$ ), and red (band around $620 \mathrm{~nm}$ ). Figure 1 shows a comparison of these spectral bands [Fig. 1(c)] with the hemoglobin absorption spectra [Fig. 1(a)] and typical mucosal scattering coefficients [Fig. 1(b)]. Regarding the dependence of these spectral bands on the tissue optical parameters, the following properties are indicated: (1) since $\mu_{\mathrm{s}}^{\prime}$ increases as the wavelength decreases, the $450 \mathrm{~nm}$ blue band is particularly sensitive to the scattering property; (2) since the $470 \mathrm{~nm}$ blue band corresponds to the point where the absorption gap of oxy- and deoxyhemoglobin is locally wide, the reflectance at the $470 \mathrm{~nm}$ blue band is sensitive to $\mathrm{StO}_{2}$; (3) since the broad green band corresponds to the absorption peak, the reflectance at the green band is effectively sensitive to blood concentration; and (4) since the absorption at the red band is extremely small, the reflectance at the red band contains very little information about the tissue blood, and hence can be used as a reference standard.

The algorithm for the $\mathrm{StO}_{2}$ calculation comprises the following steps: (1) obtain the diffuse reflectance of the observed tissue at the four spectral bands, (2) calculate the ratios of the reflectance at different wavelengths, and (3) transform the ratios into the tissue characteristics $\left(\mathrm{StO}_{2}\right.$ and blood concentration). The physical principle based on a simple model of tissue optics is described below.

Optical properties of biological tissue are generally characterized by three quantities: the absorption coefficient $\mu_{\mathrm{a}}$, the scattering coefficient $\mu_{\mathrm{s}}$, and the anisotropy factor $g$. Here, we consider a tissue as a homogeneous semi-infinite slab that is vertically irradiated by homogeneous illumination. Based on scale invariance arguments on this model, the total diffuse reflectance of the tissue depends on only the ratio $\mu_{\mathrm{a}} / \mu_{\mathrm{s}}^{\prime}$, that $R(\lambda)=R\left(\mu_{\mathrm{a}}(\lambda) / \mu_{\mathrm{s}}^{\prime}(\lambda)\right)$, where $\mu_{\mathrm{s}}^{\prime}=\mu_{\mathrm{s}}(1-g)$ is the reduced scattering coefficient. ${ }^{25}$ Since the hemoglobin is the dominant absorptive component in the gastrointestinal tissue, $\mu_{\mathrm{a}}$ can be written as

$\mu_{a}(\lambda)=c_{\mathrm{Hb}}\left\{\frac{\mathrm{StO}_{2}}{100} \mu_{a \mathrm{HbO}_{2}}(\lambda)+\left(1-\frac{\mathrm{StO}_{2}}{100}\right) \mu_{a \mathrm{Hb}}(\lambda)\right\}$,

where $c_{\mathrm{Hb}}$ is the volume fraction of the blood in the tissue; $\mu_{\mathrm{aHbO} 2}$ and $\mu_{\mathrm{aHb}}$, respectively, denote the absorption coefficient of the blood of $\mathrm{StO}_{2}=100 \%$ and $0 \%$ [Fig. 1(a)]; and the $\mathrm{StO}_{2}$ is the oxygen saturation of the blood contained in the tissue. The reduced scattering coefficient of biological tissue is generally characterized by the exponential monotone decrease according

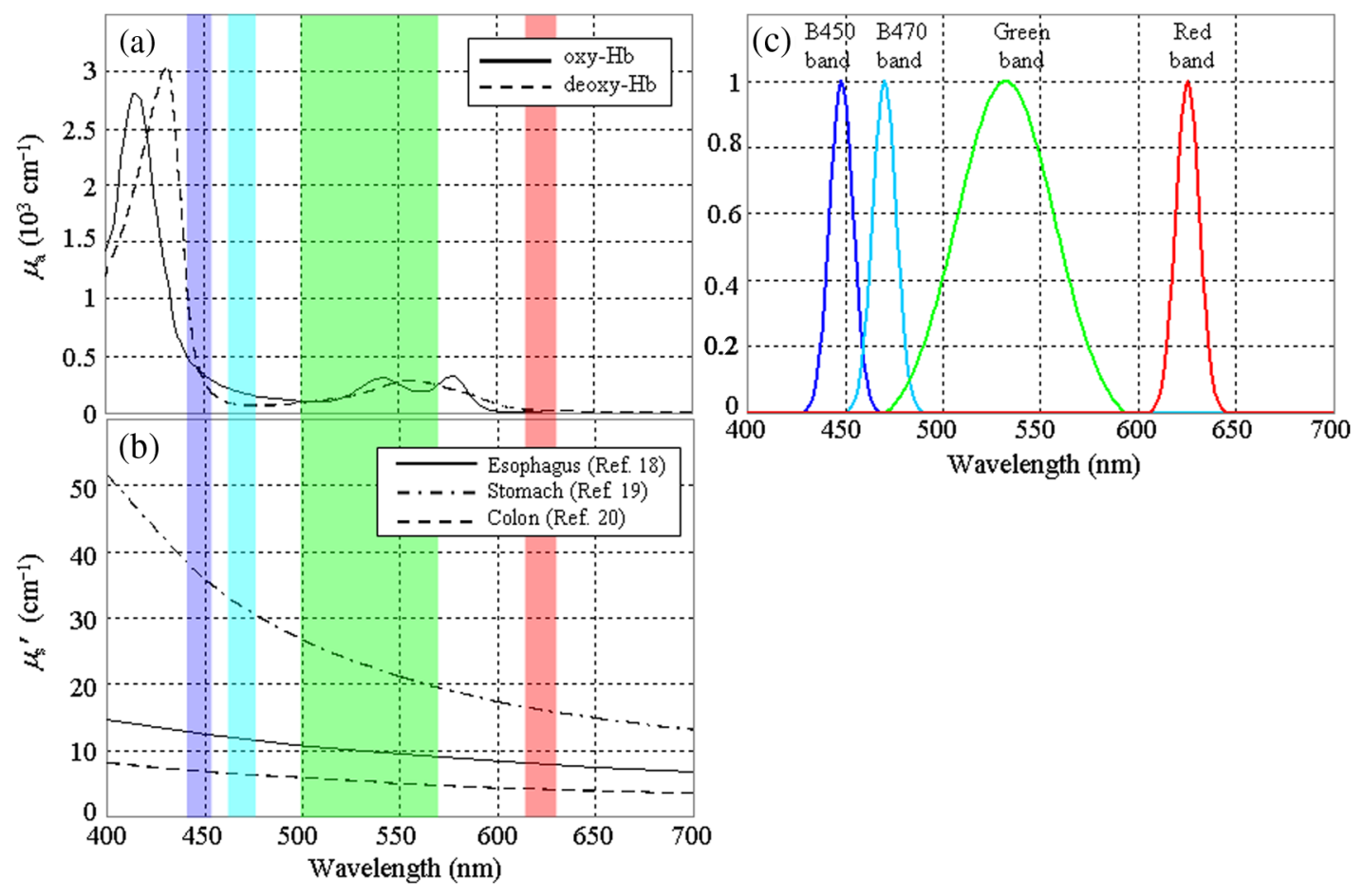

Fig. 1 (a) $\mu_{\mathrm{a}}(\lambda)$ of oxy- and deoxy-hemoglobins; (b) $\mu_{\mathrm{s}}^{\prime}(\lambda)$ of typical gastrointestinal mucosae of esophagus, ${ }^{18}$ stomach, ${ }^{19}$ and colon; ${ }^{20}$ and (c) the four spectral bands used for this technique. The spectral bands are overlaid on the graphs in (a) and (b). For the hemoglobin $\mu_{\mathrm{a}}(\lambda)$ values, we combined three literature values from Refs. $21-23$ to fully match them with actual values. ${ }^{24}$ 
to wavelength and can be modeled by the following equation with two parameters $\sigma$ and $\beta:^{26}$

$\mu_{s}^{\prime}(\lambda)=\sigma\left(\frac{\lambda}{500 \mathrm{~nm}}\right)^{-\beta}$

where $\lambda$ is normalized by $500 \mathrm{~nm}$. The parameter $\sigma$ defines the overall strength of scattering, and $\beta$ determines the slope of decrease. Using these parametrizations and the dependence on the ratio of $\mu_{\mathrm{a}} / \mu_{\mathrm{s}}^{\prime}$ by the scale invariance property as mentioned above, the diffuse reflectance $R(\lambda)$ of the tissue can be described as a function

$R(\lambda)=R\left(C, \beta, \mathrm{StO}_{2} ; \lambda\right)$,

where $C=\mathrm{c}_{\mathrm{Hb}} / \sigma . R(\lambda)$ is characterized by the three parameters $C, \beta$, and $\mathrm{StO}_{2}$.

Let $S_{\mathrm{B} 450}, S_{\mathrm{B} 470}, S_{\mathrm{G}}$, and $S_{\mathrm{R}}$ be the obtained image signals corresponding to the four spectral bands. Here, each signal $S_{i}$ ( $i=\mathrm{B} 450, \mathrm{~B} 470, \mathrm{G}$, and $\mathrm{R}$ ) can be written as

$S_{i}\left(C, \beta, \mathrm{StO}_{2}, L_{D}\right)=L_{D} \int L_{i}(\lambda) R\left(C, \beta, \mathrm{StO}_{2} ; \lambda\right) S_{\mathrm{CCD}}(\lambda) \mathrm{d} \lambda$

where $L_{i}(\lambda)$ is the illumination spectrum of each band [Fig. 1(c)], $S_{\mathrm{CCD}}(\lambda)$ is the sensitivity of the image sensor, and $L_{D}$ is the arbitrary proportionality coefficient that depends on the distance between the tissue and the image acquisition system.

To investigate the dependence of each signal $S_{i}$ on the three tissue parameters, we simulated the diffuse reflectance of the homogeneous tissue using the software package MCML, ${ }^{27}$ at various conditions of the parameters of $C, \beta$, and $\mathrm{StO}_{2}$. The details of simulation conditions are described in Sec. 4.1.4. Figures 2(a)-2(c) show the simulated parameter dependence of each signal in the $\log$ scale. These results indicate the inverse dependence of $S_{\mathrm{B} 450}$ and $S_{\mathrm{G}}$ on $\beta$ [Fig. 2(a)], the large differences in the degree of dependence of each signal on $c_{\mathrm{Hb}}$ (hence on $C$ ) [Fig. 2(b)], and the strong dependence of $S_{\mathrm{B} 470}$ on $\mathrm{StO}_{2}$. Based on the results, we consider the following three signal ratios in the $\log$ scale $\left(L_{D}\right.$ is canceled here):

$x=\log \frac{S_{\mathrm{G}}}{S_{\mathrm{B} 450}}, \quad y=\log \frac{S_{\mathrm{B} 470}}{S_{\mathrm{G}}}, \quad z=\log \frac{S_{\mathrm{R}}}{S_{\mathrm{G}}}$.

The simulated parameter dependence of each signal ratio is shown in Figs. 2(d)-2(f). These results shows the strong dependence of $x, y$, and $z$ on $\beta, \mathrm{StO}_{2}$, and $c_{\mathrm{Hb}}$, respectively.

Note that the three signal ratios have markedly different dependences on the three tissue parameters. Each signal ratio does not depend on the single tissue parameter but on the three parameters simultaneously and nonlinearly. However, if we consider the three-dimensional (3-D) space spanned by $(x, y, z)$, we can define nonlinear surfaces, each of which

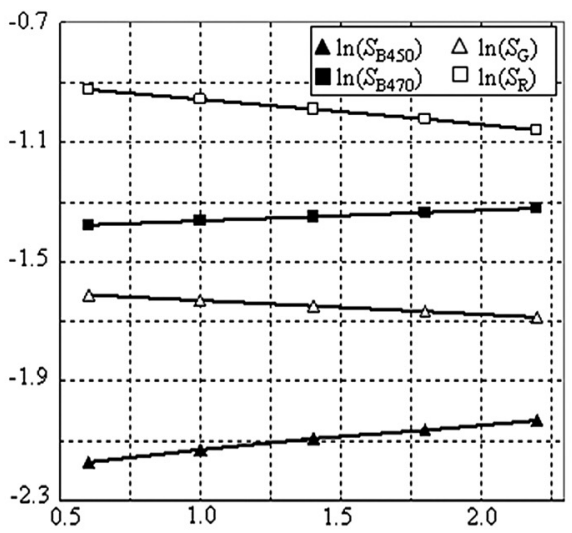

(a)

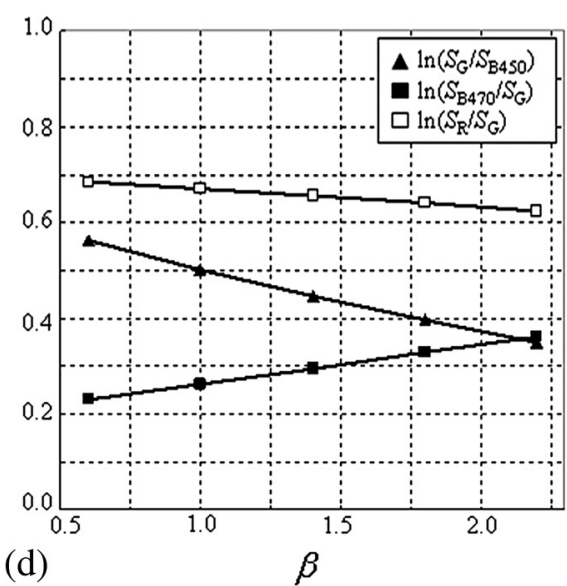

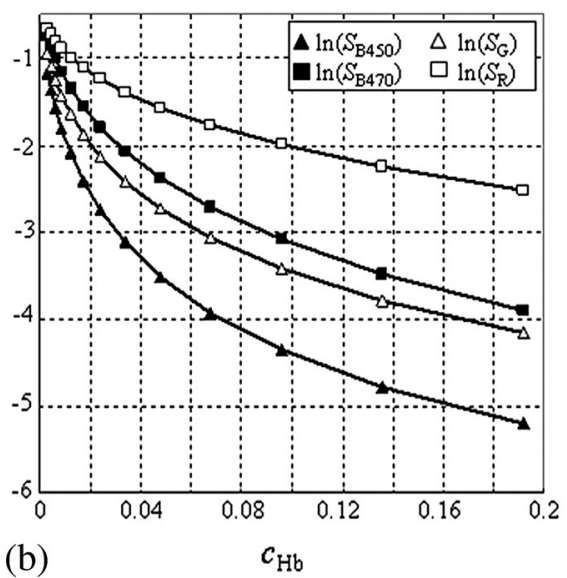

(b)

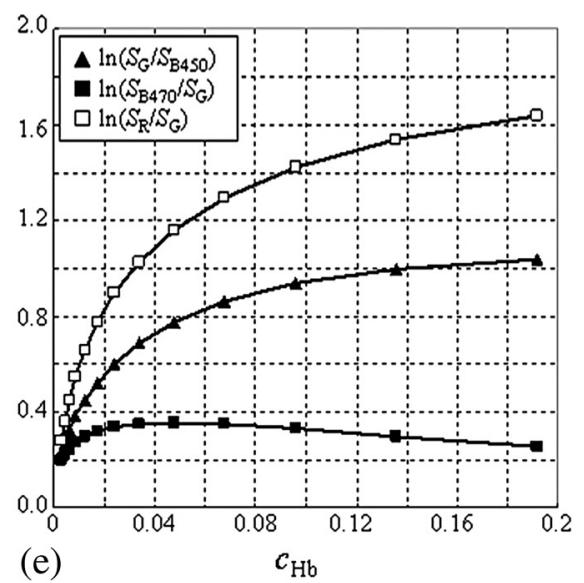

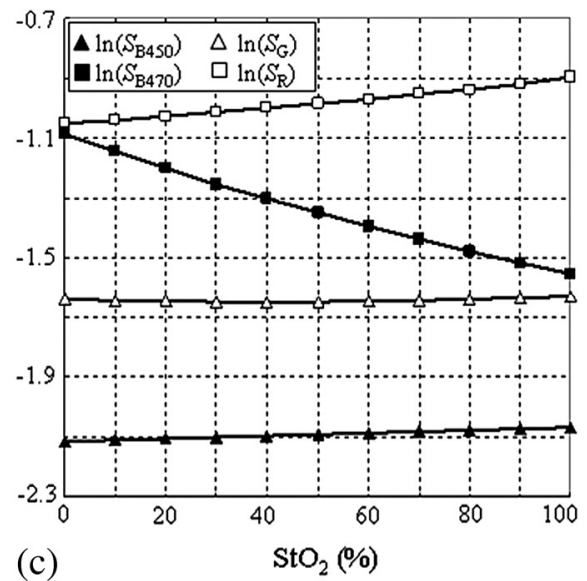

(c)

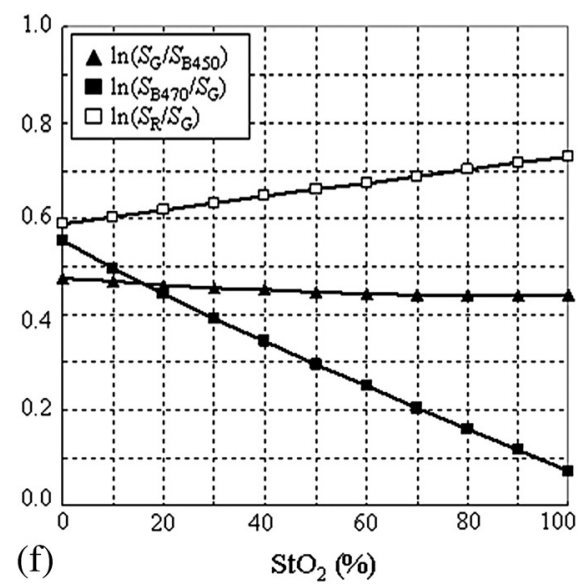

Fig. 2 (a)-(c) Simulated dependences of the four signals and (d)-(f) the three signal ratios on the tissue parameters. For these simulations presented here, only one parameter was varied while the other two were fixed at the standard values, defined as $\beta=1.4, c_{\mathrm{Hb}}=0.012, \sigma=14 \mathrm{~cm}^{-1}$, and $\mathrm{StO}_{2}=50 \%$. 
corresponds to the same $\mathrm{StO}_{2}$ value, and $\mathrm{StO}_{2}$ can be obtained separately from other two parameters. This is the principle of our technique. The details of the transformation method of $(x, y, z)$ into $\mathrm{StO}_{2}$ are described in Sec. 4.1.4.

\section{Development of $\mathrm{StO}_{2}$ Imaging Endoscope}

Based on the principle described in Sec. 2, we developed a prototype endoscope system for gastroenterological endoscopy that allowed us to obtain $\mathrm{StO}_{2}$ distribution images of tissue. As with the other types of our prototype systems, ${ }^{17,28}$ the light source of the system provided four bands of light: two blue narrow bands at 450 and $470 \mathrm{~nm}$, a green broad band, and a red band [Fig. 1(c)]. Images were acquired with an RGB color CCD (same sensor as that mounted on EG590 endoscope, Fujifilm Co., Tokyo, Japan) mounted at the endoscope tip, and the image data were transmitted to the processor and saved on the hard disk of a computer that was connected to the processor. The illumination was switched sequentially among the four bands synchronized with the video frames at a rate of 30 frames/s. Switching the illumination provided different spectroscopic images of the four colors at almost the same position on the object being observed unless the object moved quickly. The $\mathrm{StO}_{2}$ images were obtained at a rate of 7.5 frames/s in almost real time. The processing time from the data acquisition to display on the monitor was about $0.1 \mathrm{~s}$.

\section{Experiment}

\subsection{Materials and Methods}

\subsubsection{Ethics statement}

Prior to the study, the protocol of the experiment was reviewed and approved by the Fujifilm Imaging Technology Center. As for the human blood used in this experiment, it was confirmed by Kohjin Bio Co. Ltd. that the informed consent for its research usage had been obtained by the donor.

\subsubsection{Tissue-simulating phantoms}

We prepared tissue-simulating phantoms according to the following procedure. ${ }^{8}$ Precipitated erythrocytes $(0.8 \mathrm{ml})$ were drawn from preserved human blood (Kohjin Bio Co., Ltd., Sakado, Japan). Then $4 \mathrm{ml}$ of saline was added to the erythrocytes, and the mixture was centrifuged at $1500 \times \mathrm{g}$ for 2.0 min. The supernatant was subsequently pipetted away. After the process was repeated twice, $20 \mathrm{ml}$ of distilled water was added to hemolyze the erythrocytes completely over $15 \mathrm{~min}$. The fluid was centrifuged again, and the fluid portion was extracted to obtain the hemoglobin solution. The concentration of this solution was measured by absorptiometry, and the solution was diluted to the target concentration. We prepared three types of phantoms, whose conditions are summarized in Table 1 . We added Intralipid $10 \%$ solution as the light scatterer. A total of $125 \mathrm{ml}$ of the phantom liquid was put into a 100$\mathrm{ml}$ flask, and $0.6 \mathrm{~g}$ of sugar was added. The $\mathrm{pH}$ was then adjusted to 7.4 by adding $\mathrm{NaOH}$. The temperature of the phantom was kept at $37^{\circ} \mathrm{C}$ by putting the flask into a thermostat bath.

The following procedure was used for absorptiometric measurement of the hemoglobin concentration. Some of the hemoglobin solution was put into a glass cell, and the sample was illuminated by collimated light from a halogen lamp through a light diffuser. The spectra of the transmitted light of the sample and a blank cell were measured with a spectroradiometer
Table 1 Conditions of tissue-simulating phantoms.

\begin{tabular}{lcc}
\hline & $\begin{array}{c}\text { Blood volume } \\
\text { fraction }(\mathrm{w} / \mathrm{v} \%)\end{array}$ & $\begin{array}{c}\text { Intralipid } \\
\text { concentration }(\mathrm{w} / \mathrm{v} \%)\end{array}$ \\
\hline Type 1 & 1.0 & 0.7 \\
Type 2 & 1.0 & 1.4 \\
Type 3 & 2.0 & 0.7 \\
\hline
\end{tabular}

SR-UL1 (Topcon Technohouse Co., Tokyo, Japan), and then the transmittance spectrum of the sample was obtained. The difference in the absorbance at wavelengths of 570 and $650 \mathrm{~nm}$ was compared with literature data of an oxy-hemoglobin solution of a fixed concentration, and then the concentration was derived.

\subsubsection{Data and image acquisition}

Figure 3 shows the experimental setup. The flask containing the liquid tissue-simulating phantom was fixed in the thermostat bath. The mouth of the flask was closed with a rubber plug with holes for probes and other devices. A flexible Clarketype catheter microelectrode (RF CC1.R oxygen probe, Integra LifeSciences Co., Plainsboro) was used for $\mathrm{dO}_{2}$ measurement. The oxygen electrode was calibrated by measuring the current of the auxiliary calibration standard liquid according to the usage. The oxygen electrode, the auxiliary thermometer probe, and the tip of the endoscope were inserted into the flask through the holes of the rubber plug. The electrode and
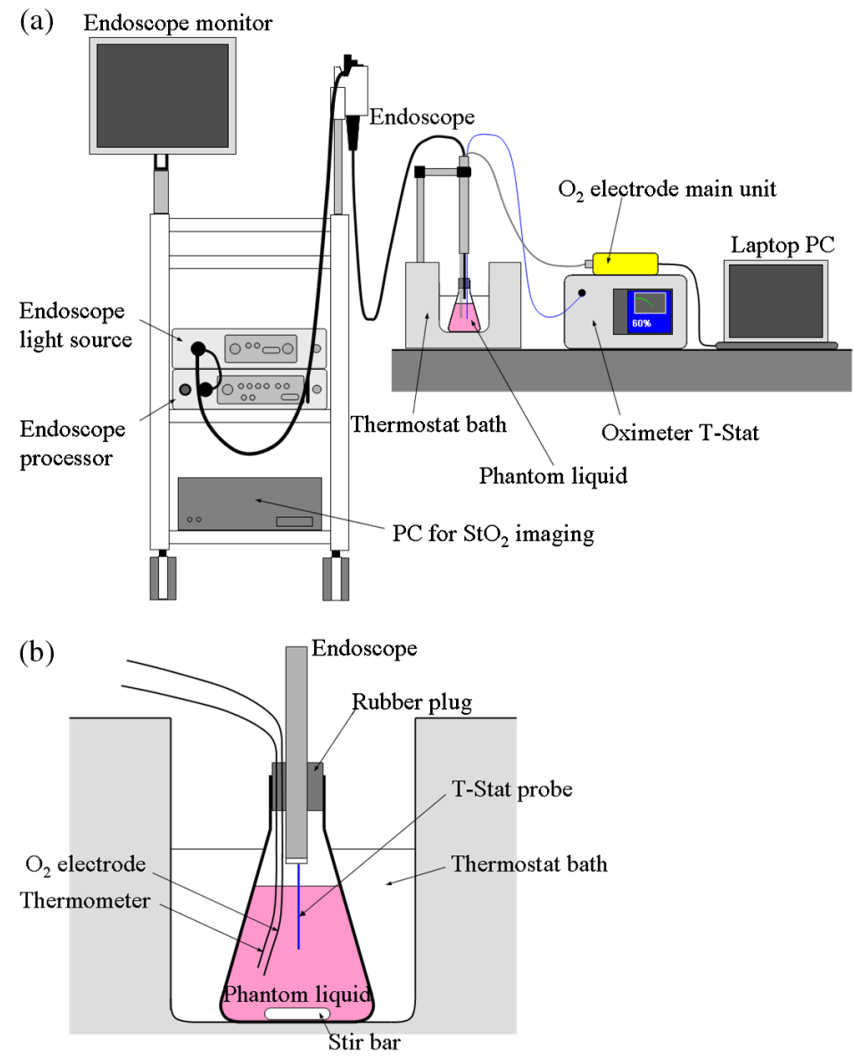

Fig. 3 Experimental setup for the tissue-simulating phantom measurements: (a) overview of the whole system and (b) the phantom in the thermostat bath. 
thermometer probe were immersed into the phantom. The tip of the endoscope was fixed $1 \mathrm{~cm}$ above the liquid level. Next, $0.3 \mathrm{~g}$ of dry yeast suspended in a small amount of the phantom liquid was put into the flask to gradually reduce the phantom $\mathrm{dO}_{2}$. The holes of the rubber plug were sealed with oil-based nonhardenable putty to keep the phantom airproof. The endoscopic sensor probe of the T-Stat tissue oximeter was inserted into the phantom through the instrumental channel of the endoscope, and the probe tip was fixed approximately $3 \mathrm{~cm}$ below the liquid level. We monitored the phantom $\mathrm{StO}_{2}$ with T-Stat. The phantom was constantly stirred during the experiment except when the images were acquired with the endoscope. Since the endoscope illumination affected the $\mathrm{StO}_{2}$ measurement with T-Stat, we turned on the illumination only when we acquired images with the endoscope. The illumination of the T-Stat probe was dark enough at the phantom liquid level that it did not affect the endoscopic images.

\subsubsection{Hemoglobin oxygen saturation}

We derived the $\mathrm{StO}_{2}$ image using a set of consecutive frames of images corresponding to the four different bands of the illumination. Let $S_{\mathrm{B} 450}, S_{\mathrm{B} 470}, S_{\mathrm{G}}$, and $S_{\mathrm{R}}$ be the obtained image signals corresponding to the four spectral bands at the same position. First, each signal of the phantom image was normalized by that of an image of the reflectance standard (WS-1-SL, Labsphere, Inc., North Sutton) to make the signal proportional to the reflectance of the phantom at the corresponding wavelength range. Next, we made three signal ratios in logarithmic form as given in Eq. (5), and then transformed the ratios into a value of $\mathrm{StO}_{2}$ using the numerical correspondence of the ratios and $\mathrm{StO}_{2}$ in the 3-D space of $(x, y, z)$ as described in Sec. 2. Performing this process for all the pixels provided the $\mathrm{StO}_{2}$ distribution image.

The transformation of $(x, y, z)$ into $\mathrm{StO}_{2}$ was performed using a lookup table, which was based on the simulated diffuse reflectance by MCML. The conditions for the simulation are summarized in Table 2. The tissue thickness was set at $5 \mathrm{~cm}$, which was thick enough to consider the tissue as semi-infinitely-deep for visible light. Using the parameters in Table 2, $\mu_{\mathrm{a}}$ and $\mu_{\mathrm{s}}^{\prime}$ were calculated by Eqs. (1) and (2), respectively.

Table 2 Simulation conditions to obtain total diffuse reflectance by MCML.

$\begin{array}{ll}\begin{array}{l}\text { Refractive index } \\ \text { Thickness }\end{array} & \begin{array}{l}1.4 \\ \mathrm{~cm}\end{array} \\ \begin{array}{l}\text { Volume fraction of the } \\ \text { blood } c_{\mathrm{Hb}} \text { in Eq. (1) }\end{array} & \begin{array}{l}13 \text { values in the range of } 0.003 \text { to } 0.192 \text { at } \\ \text { geometrically progressive intervals by a } \\ \text { factor of } 1.414\end{array} \\ \begin{array}{l}\text { Oxygen saturation } \\ \mathrm{StO}_{2} \text { in Eq. (1) }\end{array} & 0 \text { to } 100 \% \text { at } 10 \% \text { intervals } \\ \begin{array}{l}\text { Scattering coefficient } \\ \sigma \text { in Eq. (2) }\end{array} & 14 \mathrm{~cm}^{-1} \\ \begin{array}{l}\text { Wavelength exponent } \\ \beta \text { in Eq. (2) }\end{array} & 0.6 \text { to } 2.2 \text { at } 0.4 \text { intervals } \\ \begin{array}{l}\text { Anisotropy factor } g \\ \hline\end{array}\end{array}$

We calculated the total diffuse reflectance $R(\lambda)$ at each tissue condition in the wavelength range of 400 to $700 \mathrm{~nm}$ at $1 \mathrm{~nm}$ intervals, and then simulated the normalized image signals $S_{i}$ ( $i=\mathrm{B} 450, \mathrm{~B} 470, \mathrm{G}$, and $\mathrm{R}$ ) by the following equation using the sensor sensitivities and illumination spectra [corresponding to Eq. (4)] as

$$
\begin{aligned}
& S_{i}\left(C, \beta, \mathrm{StO}_{2}\right) \\
& =\sum_{\lambda=400 \mathrm{~nm}}^{700 \mathrm{~nm}} L_{i}(\lambda) R\left(C, \beta, \mathrm{StO}_{2} ; \lambda\right) S_{\mathrm{CCD}}(\lambda) / \sum_{\lambda=400 \mathrm{~nm}}^{700 \mathrm{~nm}} L_{i}(\lambda) S_{\mathrm{CCD}}(\lambda),
\end{aligned}
$$

where the summations over $\lambda$ were performed at $1 \mathrm{~nm}$ steps. The three signal ratios $(x, y, z)$ were obtained by substituting Eq. (6) into $S_{i}$ in Eq. (5).

Next, we considered the distribution of points $\mathrm{P}(x, y, z)$ in the 3-D space. Here, a combination of three tissue parameters $\left(C, \beta, \mathrm{StO}_{2}\right)$ corresponded to each $\mathrm{P}(x, y, z)$, and we found that if $\mathrm{StO}_{2}$ was fixed and the other two parameters were varied, the simulated $\mathrm{P}(x, y, z)$ distributed on a mildly curved surface. This curved surface defined the iso- $\mathrm{StO}_{2}$ surface for each $\mathrm{StO}_{2}$ value as presented in Fig. 4. Exploiting this property, we created the lookup table to transform $(x, y, z)$ into $\mathrm{StO}_{2}$ as follows. We approximated each surface by a third-degree polynomial of $x z$ as

$y\left(\mathrm{StO}_{2} ; x, z\right)=\sum_{m, n=0}^{3} a_{m n}\left(\mathrm{StO}_{2}\right) x^{m} z^{n}$,

and the determined coefficients $a_{m n}\left(\mathrm{StO}_{2}\right)$ were stored in the computer's memory. Here, suppose that the obtained signal ratios by the experiment were $\left(x_{\exp }, y_{\exp }, z_{\exp }\right)$. First, we calculated $y\left(\mathrm{StO}_{2} ; x_{\exp }, z_{\text {exp }}\right)$ values by Eq. (7) for $\mathrm{StO}_{2}=$ $0 \%-100 \%$ at $10 \%$ intervals, and searched for two consecutive $y$ values $y_{1}$ and $y_{2}$ at $\mathrm{StO}_{2}=\mathrm{StO}_{2}^{(1)}$ and $\mathrm{StO}_{2}^{(2)}$, respectively, that satisfied $y_{1} \leq y_{\exp }<y_{2}$. Then we obtained the obtained the corresponding $\mathrm{StO}_{2}$ value by a linear interpolation as

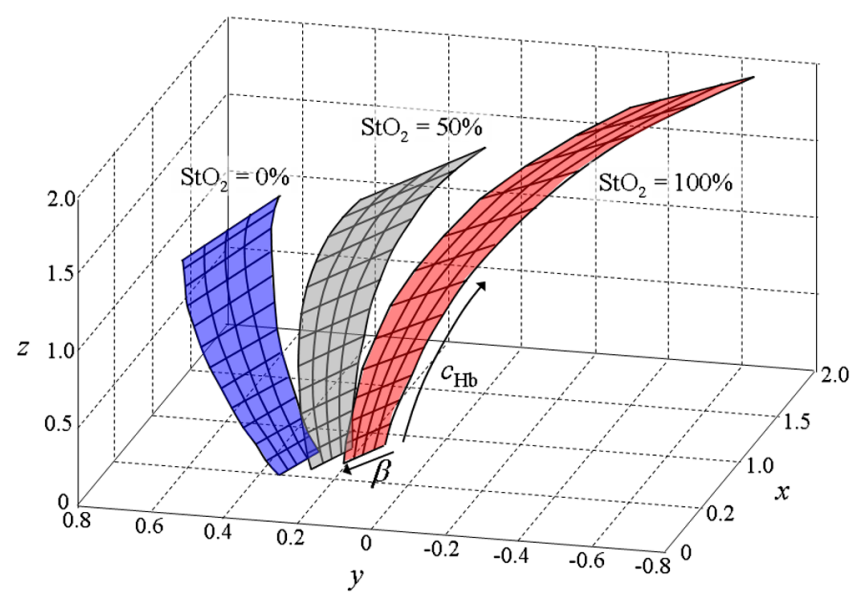

Fig. 4 Iso- $\mathrm{StO}_{2}$ surfaces in the three-dimensional space defined by the three signal ratios $(x, y, z)$. Three surfaces at $\mathrm{StO}_{2}=0,50$, and $100 \%$ are presented. Each mesh point corresponds to a simulated point. The directions corresponding to the changes in $\beta$ and $c_{\mathrm{Hb}}$ are indicated on the surface of $\mathrm{StO}_{2}=100 \%$. 


$$
\mathrm{StO}_{2 \exp }=\frac{\mathrm{StO}_{2}^{(1)}\left(y_{2}-y_{\exp }\right)+\mathrm{StO}_{2}^{(2)}\left(y_{\exp }-y_{1}\right)}{y_{2}-y_{1}} .
$$

\subsubsection{Determination of optical properties}

The optical properties of the phantom liquid were determined by the integrating sphere technique, using the same setup as described in Ref. 28. The phantom liquid was put into a custom-made planar glass cell with an inner spacing of $2 \mathrm{~mm}$, and the total transmittance $T_{t}$ and diffuse reflectance $R_{d}$ of the 2-mm-thick liquid were measured using an integrating sphere and a spectrometer. The reduced scattering coefficient $\mu_{\mathrm{s}}^{\prime}$ was determined by an inverse Monte Carlo technique. ${ }^{19}$ Here, we assumed a homogeneous, 2-mm-thick tissue model possessing the optical parameters of $\mu_{\mathrm{a}}$ and $\mu_{\mathrm{s}}^{\prime}$, and calculated its $T_{t}$ and $R_{d}$ using the software package MCML. We performed the calculation for various $\mu_{\mathrm{a}}$ and $\mu_{\mathrm{s}}^{\prime}$, and determined the optical parameters that reproduced the measured values with minimal errors.

\subsection{Results}

\subsection{1 $\mathrm{StO}_{2}$ images}

Figure 5 shows endoscopic images of the phantom liquid surface obtained at different $\mathrm{StO}_{2}$ conditions. We defined a region of interest on the phantom surface area for each image to eliminate inappropriate areas, such as specular reflection spots and the T-Stat probe. We then obtained the average $\mathrm{StO}_{2}$ value in the region of interest. Figure 6 shows the results of the phantom measurements, where $\mathrm{StO}_{2}$ measured with the endoscope and T-Stat are plotted against $\mathrm{dO}_{2}$. The literature values of the hemoglobin dissociation curve are also presented. Here, the dissociation curve was created using the parameters of Ref. 22 at physiologic conditions. The endoscopic $\mathrm{StO}_{2}$ values agree with those from the literature within $10 \%$. The raw T-Stat $\mathrm{StO}_{2}$ values disagreed in the range of $\mathrm{dO}_{2}>80 \mathrm{mmHg}$ and $<10 \mathrm{mmHg}$, where the literature $\mathrm{StO}_{2}$ becomes nearly $100 \%$ and $0 \%$, respectively. The raw T-Stat $\mathrm{StO}_{2}$ tended to show constant values at these $\mathrm{dO}_{2}$ ranges. Hence, we normalized the T-Stat $\mathrm{StO}_{2}$ values so that their saturated values at high and low $\mathrm{dO}_{2}$
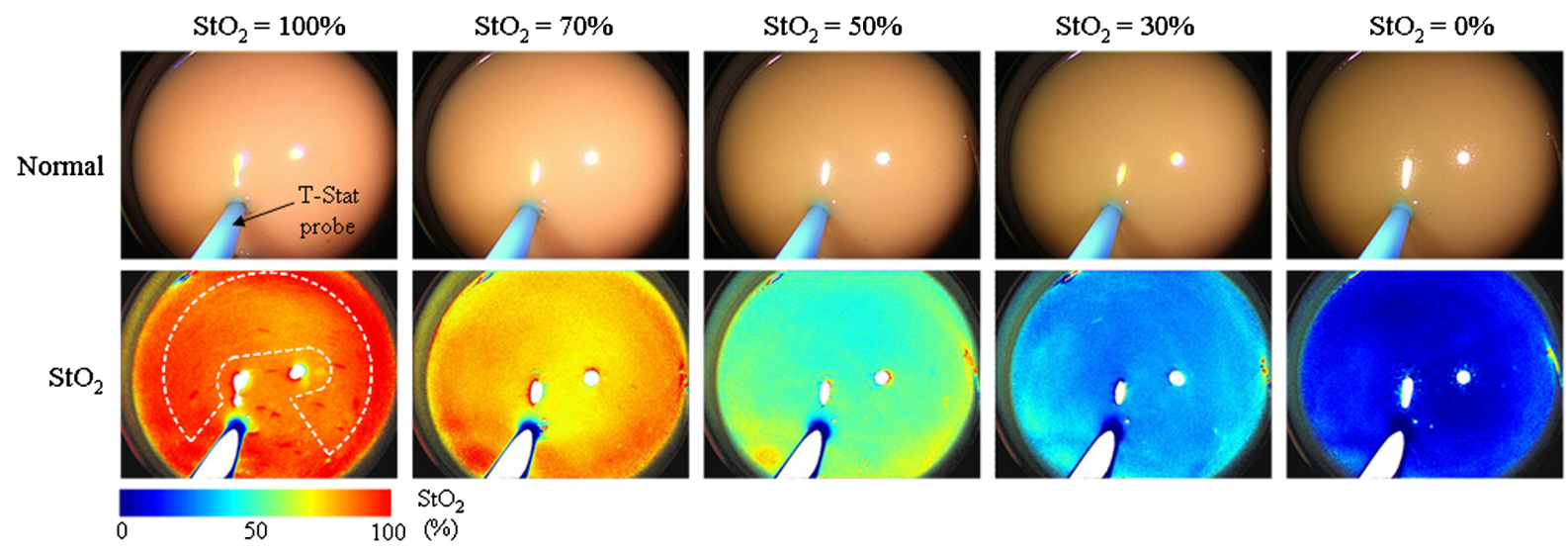

Fig. 5 Endoscopic images of the phantom liquid surface. Normal and pseudocolor $\mathrm{StO}_{2}$ images are juxtaposed for each $\mathrm{StO}_{2}$ level. The defined region of interest to calculate the average $\mathrm{StO}_{2}$ is indicated by a dashed closed curve on the $\mathrm{StO}_{2}$ image at $\mathrm{StO}_{2}=100 \%$ (the region is common to all the images).

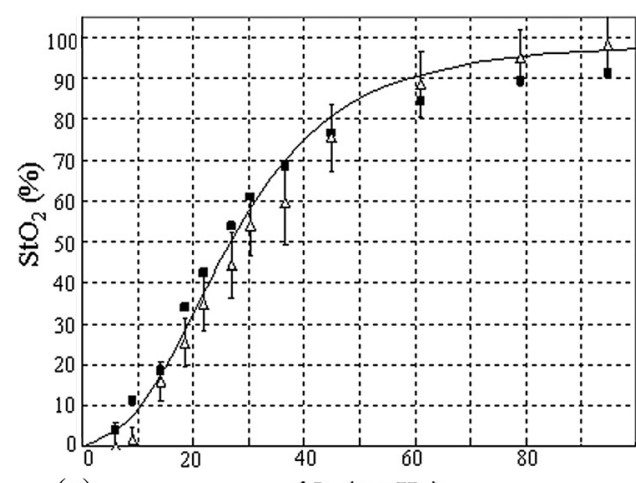

(a)
$\mathrm{dO}_{2}(\mathrm{mmHg})$

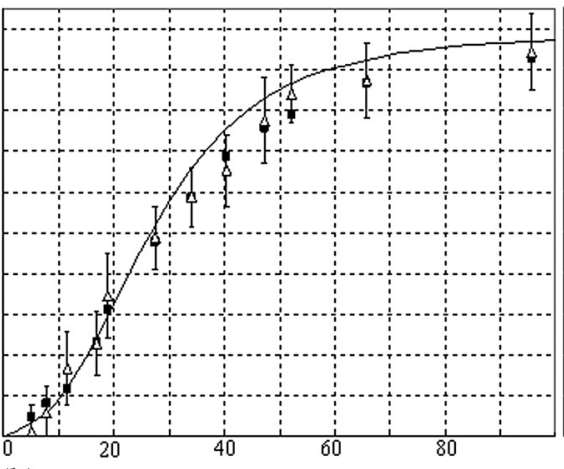

(b)

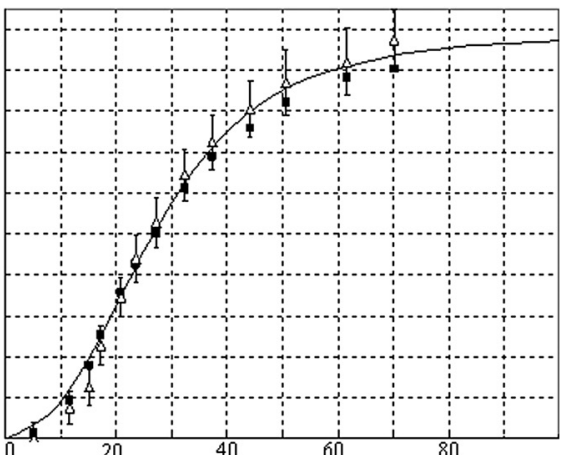

(c)
$\mathrm{dO}_{2}(\mathrm{mmHg})$

Fig. 6 Relation of $\mathrm{StO}_{2}$ (endoscope and T-Stat) with $\mathrm{dO}_{2}$ (oxygen electrode) for each type of phantom. The hemoglobin dissociation curve at physiological conditions is also plotted (solid curve). The error bars show the standard deviations of the $\mathrm{StO}_{2}$ of the endoscope in the region as presented in Fig. 4: (a) type 1 (Intralipid: $0.7 \%$, blood: $1.0 \%$ ), (b) type 2 (Intralipid: 1.4\%, blood: $1.0 \%$ ), and (c) type 3 (Intralipid: $0.7 \%$, blood: $2.0 \%)$. 

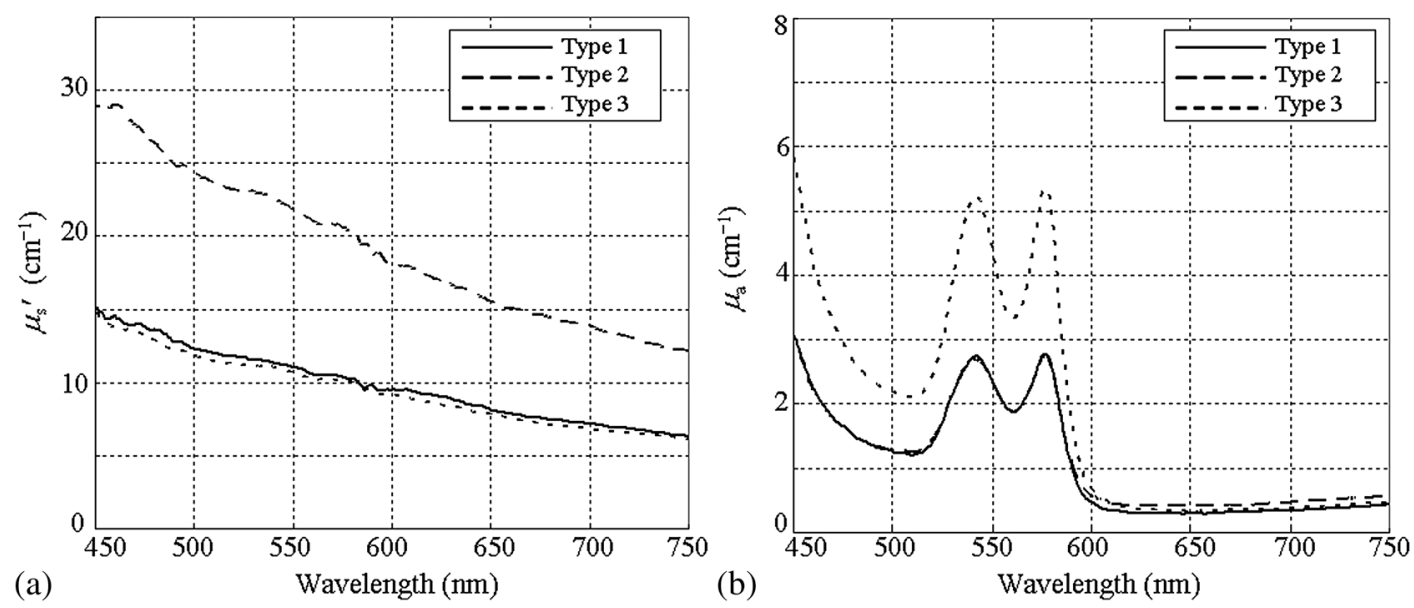

Fig. 7 Obtained optical properties of the three types of phantoms: (a) reduced scattering coefficients and (b) absorption coefficients.

became $100 \%$ and $0 \%$, respectively. The normalized values agree well with the endoscopic and literature values.

\subsubsection{Optical properties of the phantoms}

The obtained optical properties of the three types of phantoms are presented in Fig. 7, which are consistent with the phantom conditions shown in Table 1.

\section{Discussion}

In this work, we measured the $\mathrm{StO}_{2}$ and $\mathrm{dO}_{2}$ of each phantom with the endoscope, T-Stat, and an oxygen electrode simultaneously. We prepared three phantoms with different hemoglobin and Intralipid concentrations (Table 1). The results showed $\mathrm{StO}_{2}$ values of both the endoscope and T-Stat plotted against $\mathrm{dO}_{2}$ obtained by the oxygen electrode reproduced the physiological hemoglobin dissociation curve, and thus indicated that our method of calculating $\mathrm{StO}_{2}$ using four spectral band signals was valid in the range of tissue optical properties measured in this experiment.

In Sec. 2, we expressed the tissue reflectance [Eq. (3)] using three parameters, $\mathrm{StO}_{2}, c_{\mathrm{Hb}} / \sigma$, and $\beta$, by assuming a semi-infinite homogeneous tissue model, and showed that the $\mathrm{StO}_{2}$ value can be obtained using the numerical correspondence of the ratios and $\mathrm{StO}_{2}$ in the 3-D space of the three signal ratios $(x, y, z)$ as defined in Eq. (5). Here, the parameter $\beta$ is the wavelength exponent of reduced scattering coefficient $\mu_{\mathrm{s}}^{\prime}(\lambda)$ [Eq. (2)], which determines the degree of decrease of $\mu_{\mathrm{s}}^{\prime}(\lambda)$ as the wavelength increases. According to some models based on the theory of Mie scattering, the exponent $\beta$ is closely related to the scatterer size in tissue. ${ }^{29-32}$ The value of $\beta$ is restricted theoretically and semiempirically in the range of $0.20<\beta<4$.0. Scatterers of large sizes compared with the wavelength (e.g., cells and nuclei) give small values of $\beta$, while smaller scatterers give large $\beta$. The upper limit of $\beta=4.0$ corresponds to the case of Rayleigh scattering, where the scatter size is typically smaller than the wavelength of light by one order of magnitude (e.g., collagen and elastin fibrils). Therefore, different tissue structures or components can lead to different values of $\beta$. Presently, we are going to apply our $\mathrm{StO}_{2}$ imaging technique to endoscopy of the esophagus, stomach, and colorectum. The tissue of the stomach and colorectum is glandular epithelium, where single-layer epithelial cells align along the intricate glandular pitted structure and cover the collagen-rich lamina propria, while the esophageal tissue is stratified squamous epithelium, where a thick flat layer of stratified epithelial cells covers the lamina propria. Considering the differences in structure and components of these organs, the value of $\beta$ should be changed depending on the tissue being observed. Our method may provide an appropriate value of $\beta$ for each organ by making use of the four spectral band signals.

However, if we limit the target object to one organ and fix $\beta$ at an appropriate value corresponding to the optical properties of that organ, the $\mathrm{StO}_{2}$ can be obtained using only three spectral band signals $\left(S_{\mathrm{B} 470}, S_{\mathrm{G}}\right.$, and $\left.S_{\mathrm{R}}\right)$, namely the two signal ratios $y$ and $z$ in Eq. (5). In Ref. 17, we reported on a clinical study of $\mathrm{StO}_{2}$ imaging using a laser illumination endoscope system. The results showed that $\mathrm{StO}_{2}$ imaging could clearly distinguish neoplasia from non-neoplasia in the esophagus and colorectum. In that clinical study, $\mathrm{StO}_{2}$ was calculated using the three spectral bands of narrow $473 \mathrm{~nm}$, broad green, and broad red, fixing a parameter corresponding to $\beta$ at an organ-dependent value according to the method explained above. In regard to this point, the endoscope system presented in this work, based on the four band signals, is more advantageous since it does not need to assume a fixed parameter $\beta$, which leads to obtaining more precise $\mathrm{StO}_{2}$ values.

Recently, several spectral endoscopic imaging techniques have been developed, such as narrow band imaging ${ }^{33-35}$ and blue laser imaging. ${ }^{36-39}$ Both techniques exploit narrow-band blue light to enhance the increase in abnormal microvessels in the superficial mucosa, which is one of the characteristic morphological features of cancerous lesions. The technique of $\mathrm{StO}_{2}$ imaging described in this work, however, does not focus on enhancing such morphological features of the tissue, but aims at realizing a new realm of endoscopic functional imaging through visualizing tissue oxygenation. To achieve functional imaging using spectral images in endoscopy, it is essential to acquire plural spectral band images with high-speed switching of illumination wavelengths synchronized with the video frames. For practical use, the number of wavelengths should be as few as possible. The technique of this work provides a practical system using only four spectral bands to realize $\mathrm{StO}_{2}$ imaging in endoscopy. Compared with the single-point measurement of T-Stat, our $\mathrm{StO}_{2}$ imaging endoscopy has an obvious benefit-it enables real-time observation of $\mathrm{StO}_{2}$ distribution in a broad area of tissue. It is expected that this 
technique will be applied clinically and contribute to more detailed differential diagnosis or defining lesion boundaries in endoscopy by exploiting the functional information of tissue oxygenation.

\section{References}

1. J. M. Brown and W. R. Wilson, "Exploiting tumour hypoxia in cancer treatment," Nature Rev. Cancer 4, 437-447 (2004).

2. P. Vaupel and A. Mayer, "Hypoxia in cancer: significance and impact on clinical outcome," Cancer Metastasis Rev. 26(2), 225-239 (2007).

3. W. R. Wilson and M. P. Hay, "Targeting hypoxia in cancer therapy," Nature Rev. Cancer 11, 393-410 (2011).

4. B. F. Jordan and P. Sonveaux, "Targeting tumor perfusion and oxygenation to improve the outcome of anticancer therapy," Front. Phamacol. 3(94), 1-15 (2012).

5. H. K. Eltzschig and P. Carmeliet, "Hypoxia and inflammation," N. Engl. J. Med. 364(7), 656-665 (2011).

6. S. P. Colgan and H. K. Eltzschig, "Adenosine and hypoxia-inducible factor signaling in intestinal injury and recovery," Аппи. Rev. Physiol. 74, 153-175 (2012).

7. D. A. Benaron et al., "Design of a visible-light spectroscopy clinical tissue oximeter," J. Biomed. Opt. 10(4), 044005 (2005).

8. D. A. Benaron et al., "Continuous, noninvasive, and localized microvascular tissue oximetry using visible light spectroscopy," Anesthesiology 100(6), 1469-1475 (2004).

9. S. Friedland et al., "Measurement of mucosal capillary hemoglobin oxygen saturation in the colon by reflectance spectrophotometry," Gastrointest. Endoscopy 57(4), 492-497 (2003).

10. P. G. Maxim et al., "Optical detection of tumors in vivo by visible light tissue oximetry," Technol. Cancer Res. Treat. 4(3), 227-234 (2005).

11. D. van Noord et al., "Endoscopic visible light spectroscopy: a new, minimally invasive technique to diagnose chronic GI ischemia," Gastrointest. Endoscopy 73(2), 291-298 (2011).

12. M. R. Junttila and F. J. de Sauvage, "Influence of tumour micro-environment heterogeneity on therapeutic response," Nature 501, 346-354 (2013).

13. B. S. Sorg et al., "Hyperspectral imaging of hemoglobin saturation in tumor microvasculature and tumor hypoxia development," J. Biomed. Opt. 10(4), 044004 (2005).

14. K. J. Zuzak et al., "Visible reflectance hyperspectral imaging: characterization of a noninvasive in vivo system for detecting tissue perfusion," Anal. Chem. 74, 2021-2028 (2002).

15. D. N. Nakamura et al., "Automated spectroscopic imaging of oxygen saturation in human retinal vessels," Proc. SPIE 7163, 71631N (2009).

16. K. P. Nadeau et al., "Quantitative assessment of renal arterial occlusion in a porcine model using spatial frequency domain imaging," Opt. Lett. 38(18), 3566-3569 (2013).

17. K. Kaneko et al., "Hypoxia imaging endoscopy equipped with laser light source from preclinical live animal study to first-in-human subject research," PLoS One 9(6), e99055 (2014).

18. P. R. Bargo et al., "In vivo determination of optical properties of normal and tumor tissue with white light reflectance and an empirical light transport model during endoscopy," J. Biomed. Opt. 10(3), 034018 (2005).

19. A. N. Bashkatov et al., "Optical properties of human stomach mucosa in the spectral range from 400 to $2000 \mathrm{~nm}$ : Prognosis for gastroenterology," Med. Laser Appl. 22(2), 95-104 (2007).
20. H. Ao et al., "Thermal coagulation-induced changes of the optical properties of normal and adenomatous human colon tissues in vitro in the spectral range 400-1100 nm," Phys. Med. Biol. 53, 21972206 (2008).

21. S. Prahl, "Optical absorption of hemoglobin," http://omlc.org/spectra/ hemoglobin/ (March 2015).

22. W. G. Zijlstra, A. Buursma, and O. W. van Assendelft, Visible and Near Infrared Absorption Spectra of Human and Animal Haemoglobin, VSP BV, Netherlands (2000).

23. O. W. van Assendelft, Spectrophotometry of Haemoglobin Derivatives, Royal Van Gorcum Ltd., Netherlands (1970).

24. A. Amelink, T. Christiaanse, and H. J. C. M. Sterenborg, "Effect of hemoglobin extinction spectra on optical spectroscopic measurements of blood oxygen saturation," Opt. Lett. 34(10), 1525-1527 (2009).

25. G. Zonios and A. Dimou, "Modeling diffuse reflectance from semiinfinite turbid media: application to the study of skin optical properties," Opt. Express 14(19), 8661-8674 (2006).

26. S. L. Jacques, "Optical properties of biological tissues: a review," Phys. Med. Biol. 58, R37-R61 (2013).

27. L. Wang, S. L. Jacquesa, and L. Zheng, "MCML-Monte Carlo modeling of light transport in multi-layered tissues," Comput. Methods Prog. Biomed. 47(2), 131-146 (1995).

28. H. Yamaguchi et al., "Quantitative study on appearance of microvessels in spectral endoscopic imaging," J. Biomed. Opt. 20(3), 036005 (2015).

29. J. R. Mourant et al., "Predictions and measurements of scattering and absorption over broad wavelength ranges in tissue phantoms," Appl. Opt. 36(4), 949-957 (1997).

30. A. M. K. Nillson et al., "Changes in spectral shape of tissue optical properties in conjunction with laser-induced thermotherapy," Appl. Opt. 37(7), 1256-1267 (1998).

31. M. Hunter et al., "Tissue self-affinity and polarized light scattering in the Born approximation: a new model for precancer detection," Phys. Rev. Lett. 97, 138102 (2006).

32. M. Xu and R. R. Alfano, "Fractal mechanisms of light scattering in biological tissue and cells," Opt. Lett. 30(22), 3051-3053 (2005).

33. P. Lukes et al., "Narrow band imaging (NBI)—endoscopic method for detection of head and neck cancer," in Endoscopy, S. Amornyotin, Ed., pp. 75-87, InTech, Croatia (2013).

34. V. Subramanian and K. Ragunath, "Advanced endoscopic imaging: a review of commercially available technologies," Clin. Gastroenterol. Hepatol. 12(3), 368-376 (2014).

35. M. Song and T. L. Ang, "Early detection of early gastric cancer using image-enhanced endoscopy: current trends," Gastrointest. Intervention 3(1), 1-7 (2014).

36. Y. Morimoto et al., "Development of a new generation endoscope system with lasers 'LASEREO'," Fujifilm Res. Dev. 58, 1-6 (2013).

37. H. Osawa and H. Yamamoto, "Present and future status of flexible spectral imaging color enhancement and blue laser imaging technology," Dig. Endoscopy 26(1), 105-115 (2014).

38. N. Yoshida et al., "The ability of a novel blue laser imaging system for the diagnosis of invasion depth of colorectal neoplasms," $J$. Gastroenterol. 49, 73-80 (2014).

39. N. Yoshida et al., "Ability of a novel blue laser imaging system for the diagnosis of colorectal polyps," Dig. Endoscopy 26(2), 250-258 (2014).

Biographies for the authors are not available. 